\title{
Aprendizaje Basado en Competencias Aplicado a una Asignatura de Transferencia de Calor
}

\author{
Alejandro Regalado-Méndez ${ }^{(1)}$, Ever Peralta-Reyes ${ }^{(2)}$ y Juan G. Báez-González ${ }^{(3)}$ \\ (1) Instituto de Industrias, Ingeniería Ambiental, Univ. del Mar, Campus Puerto Ángel, Ciudad \\ Universitaria S/N, km 1.5 Carretara Puerto Ángel-Zipolite, Puerto Ángel, 70902, Oaxaca-México \\ (e-mail: alejandro.regalado33@gmail.com) \\ (2) Instituto de Ecología, Ingeniería Ambiental, Universidad del Mar Campus Puerto Ángel, Ciudad \\ Universitaria S/N, km 1.5 Carretara Puerto Ángel-Zipolite, Puerto Ángel, 70902, Oaxaca-México. \\ (3) Depto. Ciencia de Alimentos, Facultad de Ciencias Biológicas, Univ. Autónoma de Nuevo \\ Léon, AV. Univ. S/N Ciudad Universitaria San Nicolás de los Garza, 66451, Nuevo león-México.
}

Recibido Ene. 14, 2011; Aceptado Ene. 27, 2011; Versión final recibida Feb. 02, 2011

\begin{abstract}
Resumen
En este trabajo se diseñó una metodología de aprendizaje basado en competencias, que consta de un crucigrama de horizontales y verticales, en el se encuentran conceptos básicos de termodinámica, principios de la transferencia de calor, teoría general de sistemas y ecuaciones diferenciales. También se diseñó un cuestionario para evaluar la actitud y conocimientos de los estudiantes. Este consiste en tres tipos de autoevaluación, homogénea y heterogénea. Se encontró que la mayoría de los estudiantes demostraron buena actitud y mejoraron la adquisición de conocimientos. Se concluye que la metodología es apropiada para aumentar la comprensión global en el proceso de enseñanza-aprendizaje de los estudiantes.
\end{abstract}

Palabras clave: aprendizaje basado en competencias; teoría general de sistemas; transferencia de calor.

\section{Competency-Based Learning Applied to a Heat Transfer Course}

\begin{abstract}
A learning methodology based on competences was designed. The method consists of a horizontal and vertical crossword in which basic concepts of thermodynamic, principles of heat transfer, general systems theory and differential equations are included. Also, a questionnaire to assess attitudes and knowledge of students was designed. This consists of self-assessment, homogeneous-assessment and heterogeneous- assessment. It was found that most students had positive attitudes and improved knowledge acquisition. It is concluded that the methodology is appropriate for increasing global understanding in the teaching-learning process of students.
\end{abstract}

Keywords: competency-based learning, general systems theory, heat transfer 


\section{INTRODUCCIÓN}

Viendo el aprendizaje desde un punto de vista más profundo, no es más que buscar que el "entendimiento" se adquiera de forma clara, precisa, por lo que este puede ser adquirido por nuevos caminos. Si reemplazamos aprendizaje por "ver, conceptualizar o experiencia", observamos que hay distintos métodos de adquirir conocimientos porque aprendizaje también puede significar adquisición de "hechos o habilidades"( Baille y More 2004).

Las metodologías de enseñanza han ido cambiando en todo en el mundo, incluso en la Educación en Ingeniería. Los educadores quieren que sus estudiantes desarrollen las capacidades analíticas y pensamiento autónomo y crítico. Sin embargo, se enfrentan, muy a menudo, las dificultades derivadas de las estrategias adoptadas en el nivel de educación básica, y también de la diversidad de fondos que los estudiantes tienen cuando se inscriben a una licenciatura de una universidad (Rasteiro et al. 2009). En todos los sectores se habla de la competencia, este es un factor determinante tanto como estudiante y como trabajador. Competencia no es más que una combinación de destrezas, habilidades y conocimientos necesarios para realizar una tarea (USDE, 2001). Por lo tanto, el aprendizaje basado en competencias, es el resultado de la integración de experiencias de aprendizaje, donde las habilidades, destrezas y conocimientos interactúan para formar un nuevo conocimiento, de tal forma que cumplen con la tarea para el cual fue diseñado (Voorhees, 2001; Walter, 2000).

En México esta metodología no es muy empleada en la educación pública a diferencia de lo que se puede encontrar en Europa y Estados Unidos. En este trabajo los autores proponemos mejorar el proceso de Enseñanza-Aprendizaje (E-A) en la Universidad del Mar, de tal manera que mejoren los procesos cognitivos en los estudiantes, para formar estudiantes críticos y no procedimentales ( Regalado, et al. 2009). En este trabajo se propone una herramienta a los educadores del área de ingeniería al introducir la metodología del aprendizaje basado en competencias en la asignatura de transferencia de calor para ayudar al proceso de enseñanza aprendizaje de los alumnos que estudian una ingeniería. Esta metodología fue aplicada a alumnos de carrera de ingeniería ambiental de la Universidad del Mar, esto es con el fin de que estos mejoren sus habilidades en cuanto al análisis de problemas y resolución de problemas, gestión de proyectos y liderazgo, capacidad de análisis y pensamiento crítico, la difusión y la comunicación, las competencias interdisciplinarias, la comunicación intercultural, la innovación y la creatividad (Kuru, 2007).

\section{METODOLOGÍA}

Se diseñó un crucigrama de horizontales y verticales, en el cual se encuentran conceptos básicos de termodinámica, principios de la transferencia de calor, teoría general de sistemas y ecuaciones diferenciales. Se pretende que los alumnos contesten el crucigrama en equipos para generar competencia, pero las respuestas deben ser analizadas y explicadas en forma detallada.

\section{Evaluación de estudiante}

Es importante la evaluación de la actividad, esta será en tres etapas, auto evaluación, evaluación homogénea y heterogénea. En la autoevaluación los estudiantes deben de contestar a cada una de las preguntas que se encuentran en la Tabla 1 , de tal forma que ellos mismos pueden identificar sus destrezas, habilidades y conocimientos. Mientras que la evaluación homogénea sus compañeros le dan una calificación después de haber contestado el ejercicio, de esta forma sus mismos compañeros ayudan en la formación de sus compañeros indicándoles en donde tendrán que poner más énfasis y empeño en el proceso de aprendizaje. Por último el facilitador debe de evaluar como desempeño de cada uno de los estudiantes la actitud y conocimientos adquiridos así como aquellos adoptados en cursos anteriores. Al finalizar en cada tipo de evaluación se deben de asignar una calificación entre 0 y 10. (Duch et al.2001; Harris y Briscoe-Andrews, 2008; Yang y Wang, 2008). En la Figura 1 se presenta el crucigrama. 


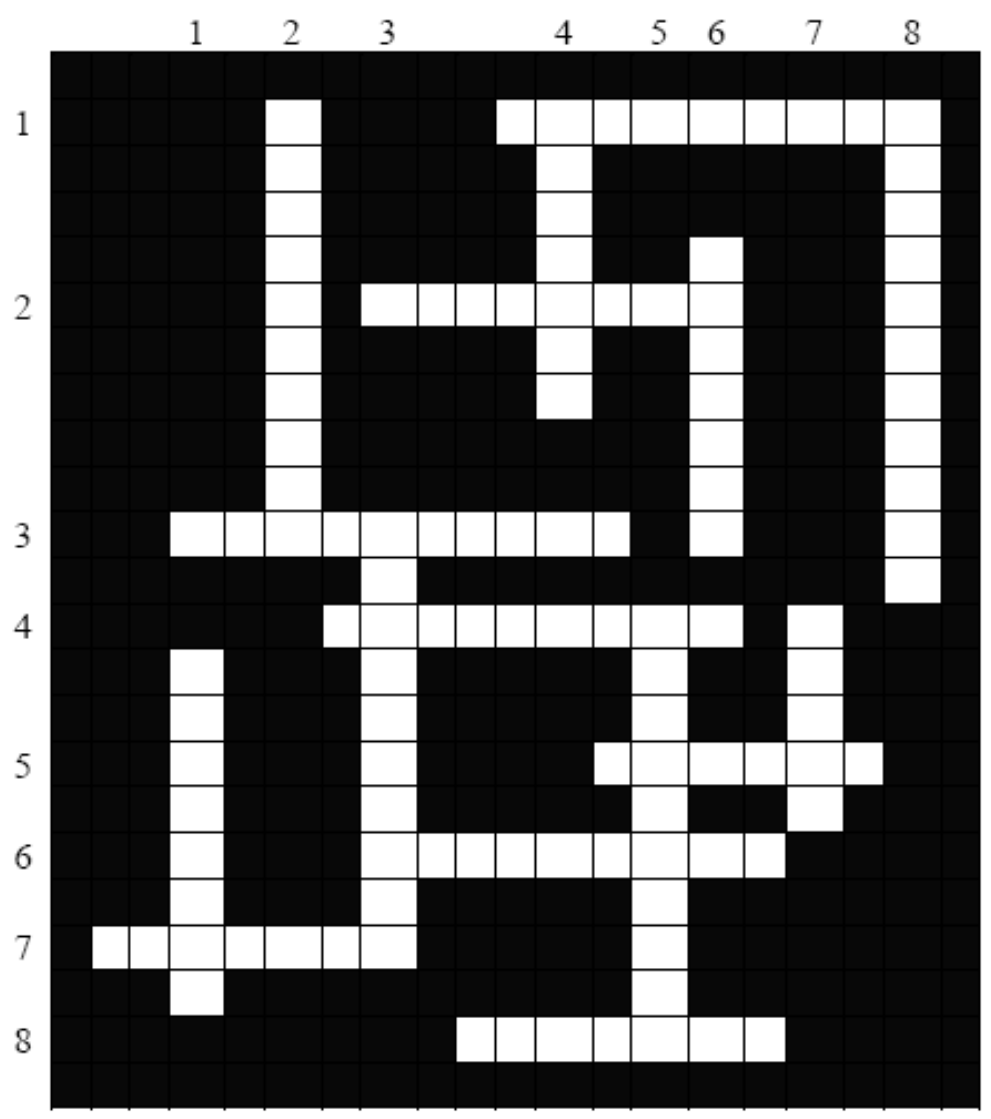

Fig. 1: Crucigrama de horizontales y verticales propuesto

\section{Horizontales}

1. Uno de los elementos de un sistema.

2. La primera ley de la termodinámica indica que la energía.

3. Mecanismo de transferencia de calor debido al movimiento molecular aleatorio más la transferencia de energía debido al movimiento global de las moléculas.

4. Nombre de la propiedad termodinámica que representa el equilibrio de fases.

5. Ley que relaciona al equilibrio líquido-vapor.

6. Mecanismo de transferencia de calor debido a las ondas electromagnéticas.

7. El potencial químico es una propiedad termodinámica usada para representar al equilibrio.

8. Nombre de la ecuación que relaciona el flux conductivo con el gradiente de temperatura en una dirección especifica.

\section{Verticales}

1. Propiedad que dice si un proceso es espontáneo en ese sentido.

2. Mecanismo de transferencia de calor debido al movimiento molecular aleatorio.

3. Si dos cuerpos que inicialmente están a distintas temperaturas, se juntan después de un intervalo de tiempo están en.

4. Nombre los sistemas que intercambian información con los alrededores.

5. Nombre de las fronteras empleadas en los modelos de entrada-salida.

6. Un globo tiene fronteras.

7. Unidad de energía en el Sistema Internacional.

8. Uno de los elementos del universo. 
Tabla 1. Preguntas para la autoevaluación de los estudiantes

\begin{tabular}{|c|c|c|}
\hline \multicolumn{3}{|l|}{ Actitud } \\
\hline 1. Si no entiendo nada cuando comienzo a resolver el problema, trato de entenderlo. & Si & No \\
\hline 2. He sido honesto con mi aprendizaje (No copio y hago mi tarea sin ayuda). & Si & No \\
\hline $\begin{array}{l}\text { 3. He sido responsable con mi aprendizaje (Busco información, siempre veo todo } \\
\text { profundamente, nunca paro por información, ni dependo de otros para hacer mi } \\
\text { trabajo). }\end{array}$ & Si & No \\
\hline 4. Hice todas las actividades indicadas. & Si & No \\
\hline 5. Participó activamente en mi equipo y contribuyo con ideas personales. & Si & No \\
\hline 6. He sido respetuoso con mi equipo (Decisiones tomadas, acuerdos, etc.). & Si & No \\
\hline \multicolumn{3}{|l|}{ Conocimientos } \\
\hline 7. Entendí todos los conceptos. & Si & No \\
\hline 8. Ahora puedo resolver problemas por mí mismo. & Si & No \\
\hline 9. A partir de este ejercicio, entiendo la importancia de trabajar en equipo. & Si & No \\
\hline
\end{tabular}

\section{RESULTADOS Y DISCUSIÓN}

Como resultados se muestra el análisis estadístico de las respuestas hechas por cada estudiante de las preguntas propuestas en la autoevaluación. Así como la comparación de los tres tipos de evaluación.

\section{Resultados de la metodología de evaluación}

En la Figura 2 se muestra el análisis estadístico de las respuestas a las preguntas hechas, de tal forma que se observa que la mayoría de los estudiantes demostraron buena actitud ante la metodología de aprendizaje basado en competencias. Además se observa que generaron nuevos conocimientos a partir de su background.

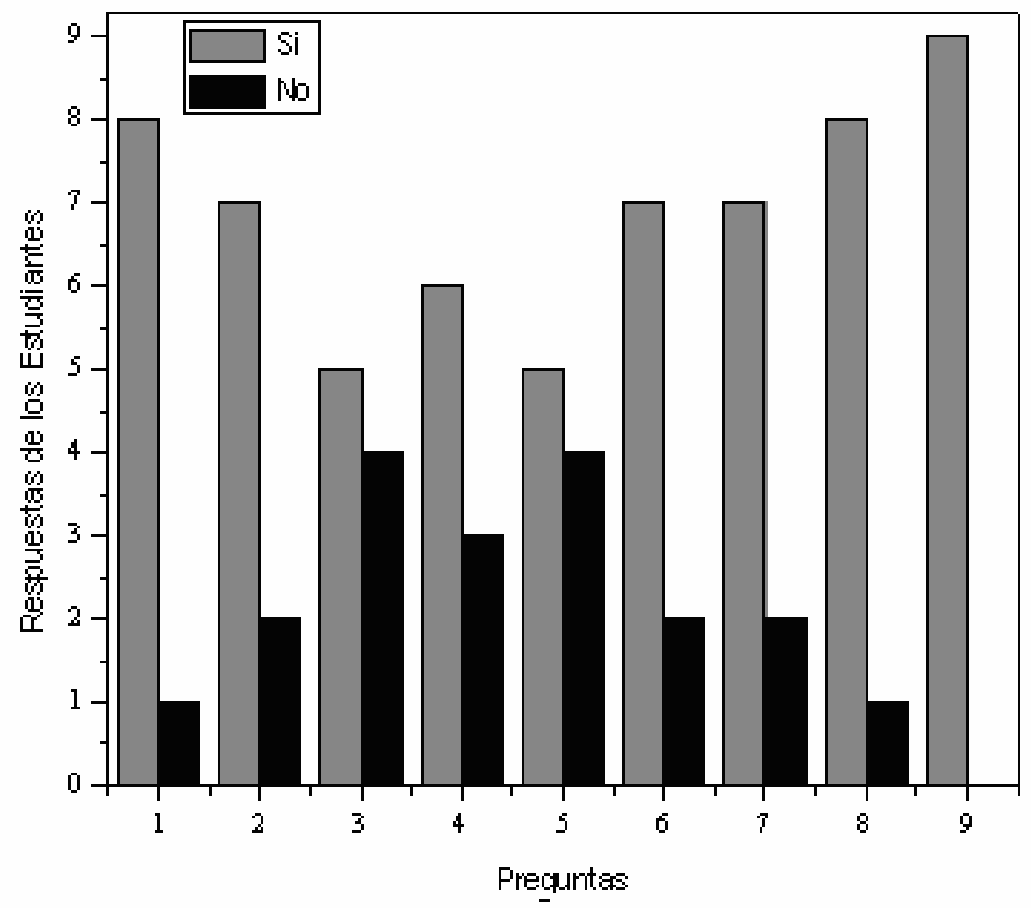

Fig. 2: Respuesta global a la evaluación 
En la Figura 3 se muestra el análisis estadístico de los tres tipos de evaluaciones, esta se observa que la actitud y los conocimientos de cada alumno fue buena, excepto por un alumno que fue el más bajo, debido a que él prefiere la enseñanza tradicional.

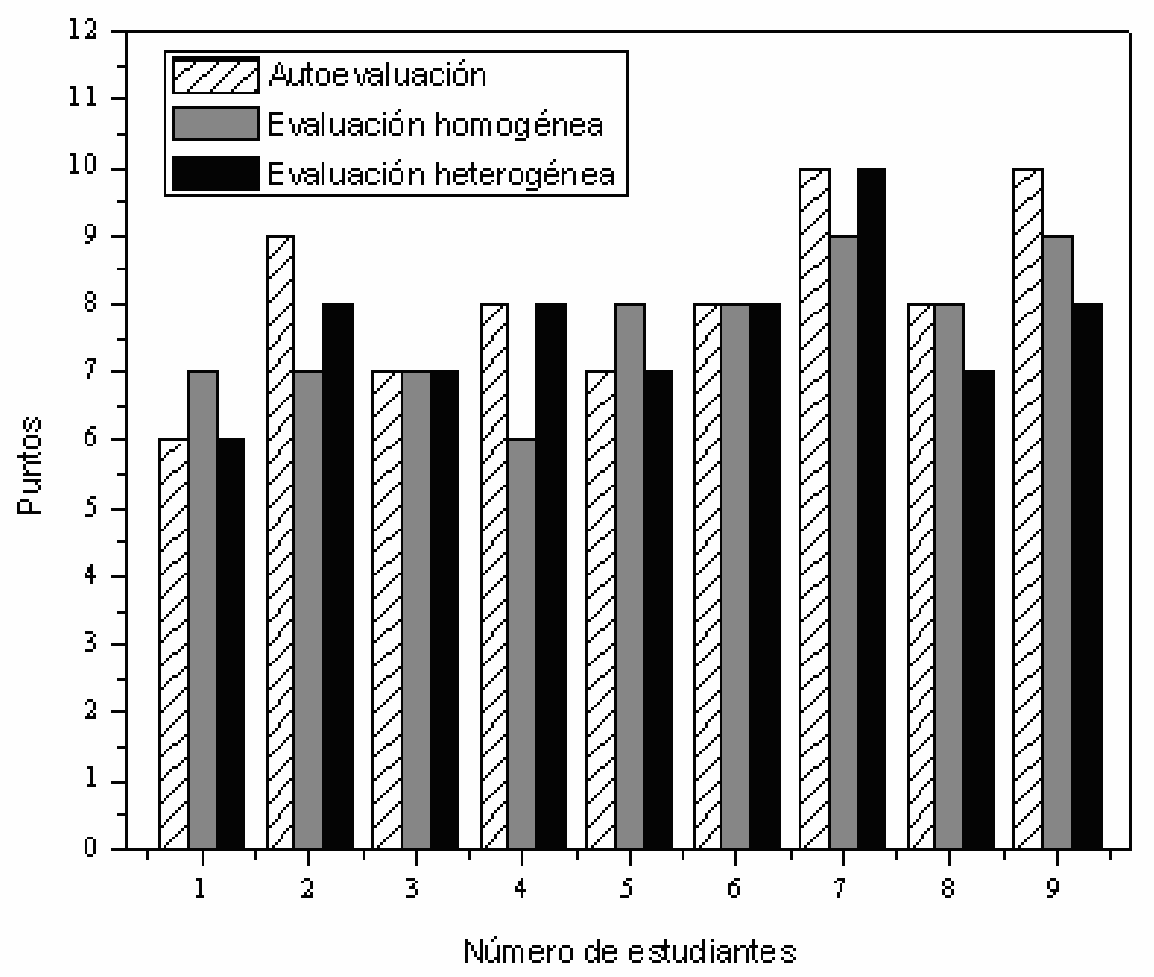

Fig. 3: Comparación de los tres tipos de evaluación

\section{Retroalimentación de alumnos}

Escuchando a los alumnos se recibe retroalimentación de su parte (Uden y Beaumont, 2006), de la cual comentaron que: "El aprendizaje basado en competencias es genial", porque:

i) el aprendizaje está centrado en los estudiantes,

ii) nos abren los ojos en la vida universitaria,

iii) ayuda a mejorar nuestras habilidades genéricas,

iv) nos hace sentir cómodos trabajando en grupo,

v) trabajar así nos hace sentir inteligentes,

vi) nos sentimos como ingenieros al resolver los problemas y

vii) la forma de abordar los problemas nos incita a saber más.

\section{CONCLUSIONES}

Una vez que los alumnos concluyeron el trabajo programado, se noto mejoría en la comprensión global del proceso cognitivo de los alumnos y en ese sentido se cumple con el objetivo de que los alumnos se apropien del conocimiento.

El método del aprendizaje basado en competencias es una herramienta fuerte para desarrollar la creatividad de los estudiantes. 


\section{REFERENCIAS}

Baille C. y More I. Effective Learning \& Teach in Engineering, $1^{\mathrm{a}}$ ed., Routle-Falmer, New York, USA (2004).

Duch B.J., Groh S.E. y Allen D.F. The Power of Problem-Based Learning, $1^{\mathrm{a}}$ ed., Sterling, Virginia, USA (2001).

Harris, A.T., y Briscoe-Andrews S. Development of a problem-based learning elective in "green engineering". Education for Chem. Eng. 3, 15-21 (2008).

Kuru, S. "Problem Based Learning", TREE - Teaching and Research in Engineering in Europe: Problem based and project oriented learning, $1^{\text {a }}$ ed., Isik University, Denmark. (2007).

Rasteiro M.G. y otros diecinueve autores. LABVIRTUAL-A virtual platform to teach chemical processes, Education for Chem. Eng. 4, 9-19 (2009).

Regalado-Méndez A. y otros cuatro autores. Problema Basado en el Aprendizaje (PBL): Encontrar los Parámetros Cinéticos Enzimáticos Integrando las Materias de Álgebra Lineal, Programación y Bioquímica, Memorias AMIDIQ XXX , 845-850. (2009).

Uden L. y Beaumont C. Technology and Problem-Based Learning, $1^{\mathrm{a}}$ ed. INFOSCI, Londres, UK (2006).

USDE, U.S. Department of Education, National Center for Education Statistics. Defining and Assessing Learning: Exploring Competency-Based Initiatives, Washington, D.C., USA (2001).

Voorhees Richard A. Competency-Based Learning Models: A Necessary Future. New Directions for Institutional Research 110, 5-13 (2001).

Walter D. Competency-based on-the-job training for aviation maintenance and inspection - a human factors approach, Int. J. of Ind. Ergonomics 26, 249-259 (2000).

Yang T., y Wang N. The Cultivation of Cluster's Sustainable Competence Based on Knowledge Management, Int. J. of Business and Management 3, 83-88 (2008). 